\title{
Numerical simulation of the target echo of radio fuse in missile-target encounter
}

\author{
Yanjie Cui ${ }^{\text {a }}$ Ziwei Wu, Man Liang, Jianguang Zhao, Wenqiang Chen and Xiangyang Zhang \\ Science and Technology on Electromagnetic Scattering Laboratory,100854Beijing, P.R. China
}

\begin{abstract}
In order to meet the requirements of fuse's digital simulation, an algorithm based on the electromagnetic scattering modelling technique of complex targets in the near field is proposed to calculate the echo power of fuse in missile-target encounter. The main research work in this paper involves the followings: (1) Considering the influence of antenna pattern and distance as well as spherical wave irradiation, a method established by the PO and PTD and GO methods is presented for calculating the pulse echo characteristics of fuse, including pulse echo power.(2) Presenting the method of modelling of near-field electromagnetic scattering in missile-target encounter. (3) Based on the theoretical analysis methods proposed in this paper, we have developed a program, and we consider a airplane model, and compute its echo signal and power under different target miss in azimuth. And the emulation results show that the average level of echo power decreases as well as the curve shift right with the increase of a miss of the target. And the echo does not change obviously under different angel of course. Numerical results prove the proposed method high efficiency. It would be especially valuable in engineering application.
\end{abstract}

Keywords: fuse; near-field; antenna Pattern; missile-target encounter.

\section{Introduction}

In military affairs domain of current domestic and overseas scholars pay a good attention on radio fuse's research and development[1,2]. And radio fuse can accurately control the real-time position of warhead explosion, which employing the warhead explosion under optimal initiating delay time as well as optimal initiating direction angle. At the same time, the efficiency target of fuse warhead matching will be obviously enhanced when using radio fuse in the weapon system.

The near-field characteristics of target echo of fuse are very important for fuse's digital simulation to enhance the efficiency target of fuse warhead matching. Nowadays, domains of current domestic and overseas researchers have focused on the study of the near-field target characteristic of the fuse, including the study on measurement and algorithm for calculating echo power of fuse. But the methods which they use to calculate the scattering characteristics of the fuse are based on the theory in the far zone, and they considered the targets as a point, and irradiating wave a plane. Inevitably there are serious specific problems for the characteristic for a near zone of location. Therefore use of principles, methods and the representations accepted for a far zone in conditions of a near zone is not

a Corresponding author : shenlinyongye@163.com

(C) 2016. The authors - Published by Atlantis Press 
obviously possible. Therefore use of principles, methods should be improved to calculate echo characteristics for fuse.

This article investigates a method based on the PO[3-5] and PTD[6] and GO[7] methods and antenna theory[8] to calculate the echo characteristics for fuse in missile-target encounter, and the proposed method would be especially valuable in engineering application.

\section{Computation of echo power of fuse and signals in the near field}

\subsection{Modeling of near-field electromagnetic scattering}

Firstly, we will introduce the basic principles of method of modeling of near-field electromagnetic scattering before the introduction of echo signals and power of fuse in missile-target encounter. The scattering field of facets and wedges is computed by Physical Optics (PO) and Physical Theory of Diffraction and multiple scattering of facet to facet is calculated by Geometrical Optics (GO) and PO.

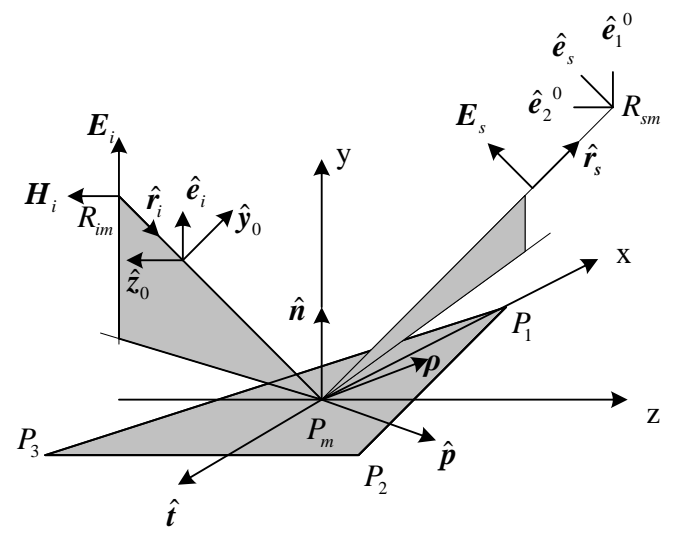

Figure 1. The system of coordinates of plane triangular element.

Referring to the method of literature [2], the electric field of irradiating wave can be represented as

$$
\boldsymbol{E}_{i m}=\sqrt{\frac{P_{i} D_{i} Z_{0}}{2 \pi}} \frac{\exp \left(i\left(k R_{i m}+\phi\right)\right)}{R_{i m}} f_{i m} \rho_{s m}
$$

Where $\mathrm{m}$ - index of elementary reflector (ER); $\boldsymbol{E}_{i m}$ - irradiating electric field of the m-th elementary reflector (ER), $k=2 \pi c / f$ - the wave number, $f_{i m}$ - values of the functions of direction of transmitting antenna in a direction on the m-th scattering element; $R_{i m}$ - distance from the beginning of system of coordinates of triangle up to the phase center of the transmitting antenna. $\rho_{s m}$ - complex coefficient of reflection of triangular element of the target, and it can be represented as:

$$
\begin{gathered}
\rho_{s m}=R_{s m} \cdot \boldsymbol{E}_{s m} / \boldsymbol{E}_{i m}=\frac{1}{4 \pi} \frac{\exp \left(i k R_{s m}\right)}{\left|\boldsymbol{q}_{\perp}\right|^{2}} \boldsymbol{T} \sum_{i=1}^{3} D_{i} \\
D_{i}=\left(\boldsymbol{q}_{\perp}^{*} \cdot\left(\hat{a}_{i+1}^{\hat{m}}-a_{i}\right)\right) \frac{\sin \left[\frac{k}{2}\left(\boldsymbol{q}_{\perp} \cdot\left(\hat{a}_{i+1}^{\hat{n}}-a_{i}\right)\right)\right]}{\frac{k}{2}\left(\boldsymbol{q}_{\perp} \cdot\left(\hat{a}_{i+1}^{\uparrow}-a_{i}\right)\right)} \exp \left[-i \frac{k}{2}\left(\boldsymbol{q}_{\perp} \cdot\left(a_{i+1}+a_{i}\right)\right)\right] \\
\hat{a}_{1}=\left\{P_{1 x}, 0,0\right\}, \quad \hat{a}_{2}=\left\{P_{2 x}, 0, P_{2 z}\right\}, \quad \hat{a}_{2}=\left\{P_{3 x}, 0, P_{3 z}\right\}, \quad \hat{a}_{4}=a_{1}
\end{gathered}
$$


Where $R_{s m}$ - distance from the beginning of system of coordinates of triangle up to the phase center of the receiving antenna, $\boldsymbol{E}_{s m}$-scattering field of the $\mathrm{m}$-th elementary reflector $(\mathrm{ER}) ; \boldsymbol{T}=\left(\hat{\boldsymbol{n}} \times \boldsymbol{H}_{\Sigma}\right)-\left[\left(\boldsymbol{n} \times \boldsymbol{E}_{\Sigma}\right) \times \boldsymbol{r}_{s}\right], \boldsymbol{E}_{\Sigma}=\boldsymbol{E}_{i m}+\boldsymbol{E}_{s m}$ represents sum of the electric field, $\boldsymbol{q}=\hat{\boldsymbol{r}}_{s}-\boldsymbol{r}_{i}$ represents incremental directed vector, $a_{1} a_{2} a_{3}$ - vectors directed from the beginning of coordinates of a triangle (Fig. 1) in its three tops (at calculation of vectors of coordinate of tops of a triangle; $P_{1} \quad P_{2} \quad P_{3}$ are set in system of coordinates of a triangle), $\boldsymbol{q}_{\perp}=\left\{q_{x}, q_{z}\right\} \quad$ - a projection of a vector $\boldsymbol{q}=\hat{\boldsymbol{r}}_{\boldsymbol{s}}-\boldsymbol{r}_{i}$ to a plane of a triangle, and $\boldsymbol{q}_{\perp}{ }^{*}=\left\{q_{z},-q_{x},\right\}$.All parameters' definition are shown in Fig.1.

The electric field of receiving wave can be represented as:

$$
\boldsymbol{E}_{\text {scat } 2}=\sum_{m=0}^{N_{\Sigma-1}} \boldsymbol{E}_{r s m}=\sqrt{\frac{P_{i} D_{i} Z_{0}}{2 \pi}} \sum_{m=0}^{N_{s-1}} f_{i m} \rho_{s m} \frac{\exp \left(i\left(k R_{i m}+\phi\right)\right)}{R_{i m} R_{s m}}
$$

The complex coefficient of reflection of sharp edges as well as double reflections can be deduced to calculate the scattering field of wedges and multiple scattering field according to formula(5).

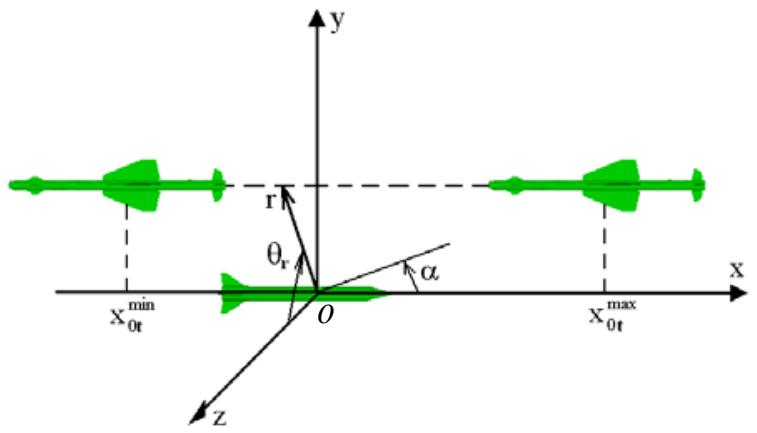

Figure 2. The trajectory of relative movement of the target in the relative system of coordinates (RSC)

\subsection{Modeling of near-field electromagnetic scattering in missile-target encounter}

Secondly, we will introduce the modeling of near-field electromagnetic scattering in missile-target encounter. The trajectory of relative movement of the target in the relative system of coordinates (RSC) represents the direct line connecting initial $P_{t 0}=\left\{x_{t 0}, y_{t 0}, z_{t 0}\right\}$ and final $P_{t k}=\left\{x_{t k}, y_{t k}, z_{t k}\right\}$ position of a conditional point of the target at its movement in parallel of an axis x of RSC (Fig. 2).

There are four systems of coordinates need to be found to calculate the near-field electromagnetic scattering in missile-target encounter: system of coordinates of relative positioning of a rocket and the aerodynamic target, system of coordinates of the target and system of coordinates of the antenna and system of coordinates of the elementary reflector (ER) in the trajectory of relative movement of the target represents the direct line. The relative system of coordinates is established to describe missiletarget encounter with parallel track, as shown in Fig. 2. In SCT calculation of intensity of a resulting field of scattering of object is carried out, and in system of coordinates of the antenna basis of the irradiating and reflected waves in a direction on ER are formed. In system of coordinates of ER calculation of intensity of a field of scattering of the reflector is carried out. For definition basis of the irradiating and reflected waves should be set in system of coordinates of ER. Then according to the formula (5), we can derive total near-field EM scattering characteristics in missile-target encounter.

Next,we deduce the formula of power characteristics of input signals of radar. For radar working in a continuous mode power of its input signal is defined from the following formula:

$$
P=\frac{\left|\dot{U}_{i n p}\right|}{2 Z_{a}}
$$


Where $\dot{U}_{\text {inp }}$ - amplitude of a voltage of an input signal, $Z_{a}$ - resistance of radiation of the antenna.

The resulting voltage in loading of the reception antenna $\dot{U}_{\text {inp }}$, arising from influence of all scattering element, forming polygonal model of object, is defined by analogy with (7) as a result of coherent summation of local voltages arising in loading of the antenna from influence of everyone $\mathrm{m}$ th scattering element:

$$
\dot{U}_{i n p}=\sum_{m=0}^{\tilde{N}_{\Sigma}-1} \dot{U}_{m}
$$

Where $\dot{U}_{m}$ - the voltage arising in loading of the antenna from influence m-th scattering element.

The voltage in loading of the reception antenna, received from influence m-th scattering element, is defined by multiplying of its intensity of an electric field of scattering (formula(5)) on the working height of the antenna:

$$
\begin{gathered}
\dot{U}_{m}=\dot{E}_{s m} h_{a} \\
h_{a}=\sqrt{\frac{D_{s} Z_{a} \lambda^{2}}{4 \pi Z_{0}}} F_{s m}
\end{gathered}
$$

Where $h_{a}$ - the module of the working height of the reception antenna, $F_{s m}$ - value of function of an direction of the reception antenna in a way from m-th scattering element., Ds- coefficient of directed action of the reception antenna in a direction of a maximum of radiation.

With use of formulas(5-9) the resulting voltage arising in loading of reception antenna from influence of the object which is taking place in a near zone of a location, is defined as follows:

$$
\begin{gathered}
\dot{U}_{\text {inp }}=\sum_{m=0}^{N_{\Sigma}-1} \dot{U}_{m}=\sqrt{\frac{P_{i} D_{i} D_{s} Z_{a} \lambda^{2}}{8 \pi^{2}}} \exp (i(\omega t+\psi)) \dot{\Sigma}_{i}^{s} \\
\dot{\Sigma}_{i}^{s}=\sum_{m=0}^{N_{\Sigma}-1} F_{i m} F_{s m} \dot{\rho}_{s m} \frac{\exp \left(i k R_{i m}\right)}{R_{i m} R_{s m}}
\end{gathered}
$$

Where $\dot{\Sigma}_{i}^{s}$ - the resulting coefficient of scattering of the object, $\dot{\rho}_{s m}$-complex coefficient of reflection of the scattering element.

As a result of substitution of expression for calculation of working value of an input voltage $\dot{U}_{\text {inp }}$ (10) in a formula (6) power of the input signal of radar, will be calculated on the basis of the following formula:

$$
P=\frac{P_{i} D_{i} D_{s} \lambda^{2}}{(4 \pi)^{2}}\left|\dot{\Sigma}_{i}^{s}\right|^{2} .
$$

Power characteristics of input signals of radar directly are connected to power characteristics of scattering of targets. In the classical theory of a radar-location as the power characteristic of scattering concept of RCS for far zone of location is used. It is obvious, that for the object which is taking place in a near zone of location when the limit $R \rightarrow \infty$ is not carried out, the classical formula cannot be used for calculation of RCS of object. In this case RCS of object we shall define from the equation of distance of a radar-location, which has the following view:

$$
P=\frac{P_{i} D_{i} D_{s} \lambda^{2} F_{i}^{2} F_{s}^{2}}{(4 \pi)^{3} R^{4}} \sigma
$$




$$
\sigma=4 \pi R^{4} \frac{\left|\dot{\sum}_{i}^{s}\right|^{2}}{F_{i}^{2} F_{s}^{2}}
$$

Where $\mathrm{P}$ - power of input signal of radar, $\mathrm{R}$ - distance between point of a target and the phase center of the antenna, $F_{i}\left(F_{s}\right)$ - value of function of direction of the transmitting (reception) antenna in a direction on a conditional point of a target, $\sigma$ - RCS in the near field in missile-target encounter, $\left|\dot{\Sigma}_{i}^{s}\right|$ - definition according to formula(11).

\section{Results and discussions}

Based on the theoretical analysis methods proposed in former chapters, we have developed a program to compute the near-field echo power and echo signal as well as RCS in the near field in missiletarget encounter. Finally, we consider a airplane model, and compute its echo signal and power under different target miss in azimuth and target miss in azimuth.

In addition, the results of pulse echo signal of the simplified airplane model are different from the transmitting pulse signal, and the pulse echo signal broadening 7 times than the transmitting pulse signal(he transmitting pulse signal is 10ns, but the echo signal is 80ns) as shown in Fig.3.

At the same time, the results of pulse echo power of the simplified airplane model change obviously under different target miss in azimuth, as shown in Fig.4. The results change obviously under different target miss in azimuth and the results show that the average level of echo power decreases with the increase of a miss of the target, as well as the curve shift right with the increase of a miss of the target.

The results of echo power of the simplified airplane model change under different angel of course, as shown in Fig.5. The results show that the echo power of fuse does not change obviously under different angel of course. We can see that the results are at the same when the angel of course is $135^{\circ}$ or $225^{\circ}$. This is because the relation position of the fuse and the simplified airplane model are at the same.

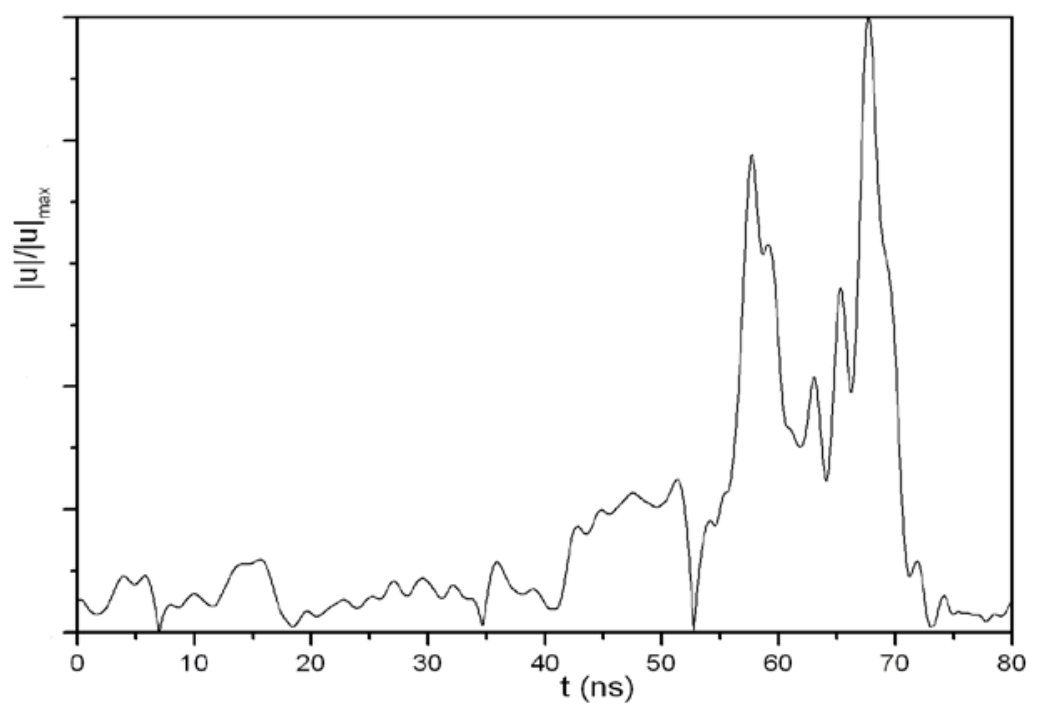

Figure 3. The echo signal of fuse of an airplane model when the transmitting signal is pulse. 


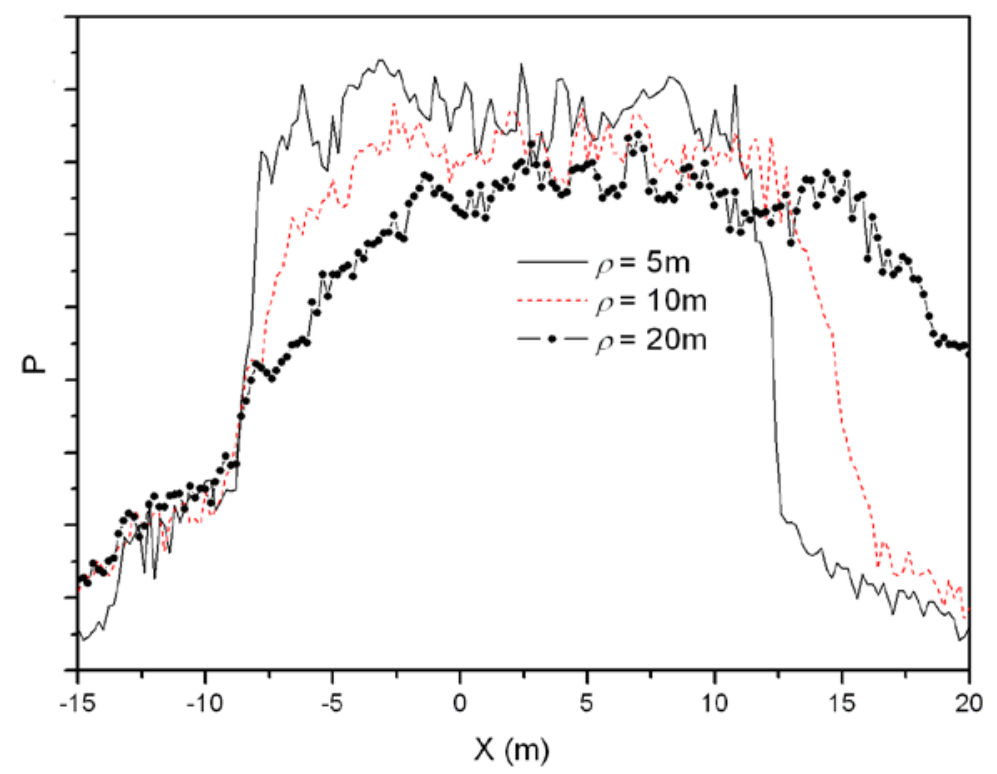

Figure 4. Compare of the echo power of fuse of an airplane model under different target miss in azimuth.

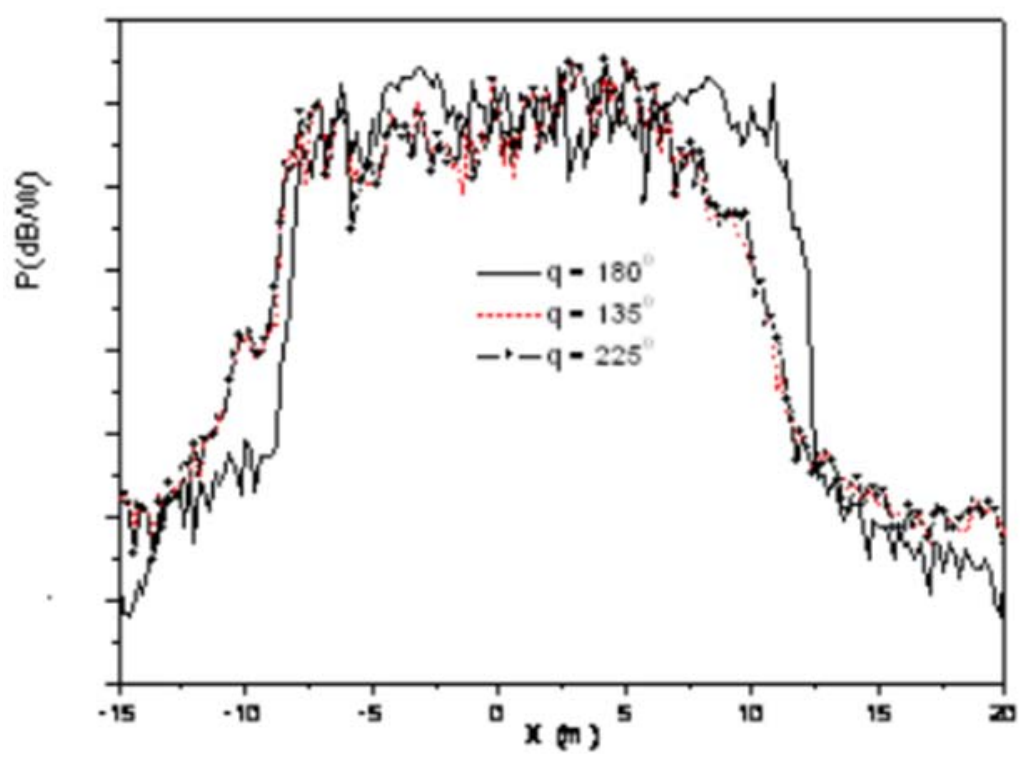

Figure 5. Compare of the echo power of fuse of an airplane model under different angel of course.

\section{Conclusion}

In conclusion, the proposed method for calculating the echo signal and power of radio fuse in this article is especially valuable in engineering application. And the emulation results show that the average level of echo power decreases as well as the curve shift right with the increase of a miss of the target. And the pulse echo signal broadens with the increase of a miss of the target. Numerical results prove the proposed method high efficiency. 


\section{References}

1. $\mathrm{Xu}, \mathrm{Xiaojian.} \mathrm{"Near-field} \mathrm{EM} \mathrm{scattering} \mathrm{calculation} \mathrm{for} \mathrm{target-seeker} \mathrm{encounter} \mathrm{simulation."} \mathrm{Proc}$ Spie 7669(2010):76691C-76691C-10.

2. Cui, Yanjie, et al. "A Fast Algorithm for Calculating Complex Targets Near-field EM Scattering Characteristics." Piers Proceedings, (2014)

3. Shyh-Kang Jeng. Near-field scattering by physical theory of diffraction and shooting and bouncing rays. IEEE Trans. on Antennas and Propagation. 46(4) pp.:551-558. (1998)

4. Huang Peikang, Yin Hongcheng, Xu xiaojian, Radar target characteristics. Electronics Industry Press, (2005)

5. N. Nazih, Youssef. Radar Cross Section of complex targets. Proceeding of the IEEE, May 77(5), (1989).

6. J .W. Crispin, and K .M Siegel. Method of Radar Cross Section Analysis. New York and London. Academic Press, (1968).

7. Wang Maoguang. The geometrical theory of diffraction (Second Edition), Xi'an Electronic and Science University press, (1994)

8. Lu Wanzheng. Antenna theory and technology, Xi'an Electronic and Science University press, (2004) 